\title{
L'Allgemeine Zeitung des Judenthums, une identité juive moderne entre intégration et auto-affirmation
}

\author{
Heidi Knörzer $^{1}$
}

\begin{abstract}
[Résumé] Depuis sa naissance au XVIIle siècle, la presse juive constitue un moteur important dans la formation d'une identité juive moderne. Dans le cas de l'Allemagne, un rôle majeur a été joué par l'Allgemeine Zeitung des Judenthums, fondé en 1837 par le rabbin Ludwig Philippson (1811-1889) et resté pendant très longtemps le journal juif le plus influent. Cet article analyse le discours identitaire de cette revue judéo-allemande et démontre qu'il s'agit d'un discours complexe qui, tout en favorisant l'intégration des Juifs à la nation, tente de contribuer à l'auto-affirmation juive. Mots-clés : Juifs allemands, presse, identité, intégration, affirmation.
\end{abstract}

[Abstract] Since its beginnings in the 18th century, the Jewish press has been a driving force in the formation of modern Jewish identity. In Germany the Allgemeine Zeitung des Judenthums played a major role in this development: founded in 1837 by the Rabbi Ludwig Philppson (1811-1889), it remained, for a significant period of time, the most influential Jewish periodical. This article proposes an analysis of identity discourse as seen in the German-Jewish journal. Our enquiry will reveal a complex discourse which attempts to contribute to Jewish self-affirmation while at the same time favours the integration of Jews into the nation.

Keywords: German Jews, the press, identity, integration, affirmation.

$\mathrm{Au}$ milieu du XVIIIe siècle, les Juifs d'Europe vivaient dans des communautés autonomes dont le mode de vie était caractérisé par l'unité que formaient la nation, la religion et les cultures juives. Cette unité commence à se défaire en même temps que se développent les processus d'émancipation politique qui ont non seulement considérablement déstabilisé la culture juive traditionnelle, mais aussi bouleversé le sentiment identitaire de la communauté. Devenue actrice de la société au même titre que les non-Juifs, la communauté a dû se construire une nouvelle identité qui tient compte d'un nouveau statut de citoyens:

«L'égalité politique exigeait un Juif de type nouveau dont l'identité serait si intimement mêlée aux modes de la société où il évolue qu'il ne serait reconnaissable que dans le cadre de cette société spécifique. Cela marque la naissance du Juif allemand, ou anglais ou français. » (Aschheim, 1982, p. 4)

1 École Polytechnique / CEREG (Paris). 
La restructuration de l'identité juive au cours de ce que l'on appelle communément "le siècle de l'émancipation » constitue un enjeu de taille. Car il s'agissait, d'une part, pour les membres des minorités juives, de formuler leur identité en des termes nouveaux, qui ne provoquent pas un affaiblissement de la culture et des traditions juives et, d'autre part, d'être reconnus comme citoyens à part entière de la nation au sein de laquelle ils vivaient, tâche rendue compliquée par une hostilité anti-juive persistante, voire croissante.

En diffusant des conceptions de lidentité juive, la presse juive qui naît au XVIIIe siècle participe de façon non négligeable à ces processus de restructuration identitaire. Dans le cas de l'Allemagne, l'Allgemeine Zeitung des Judenthums (Gazette générale du judaïsme), revue fondée en 1837 par le rabbin Ludwig Philippson (18111889), joue un rôle majeur. Dans cet article, l'objectif est de reconstruire le discours identitaire véhiculé par cette revue. Cette reconstruction se fera au moyen d'une analyse qualitative de contenu (Bardin, 1996) consistant en une lecture détaillée de tous les articles de Philippson, suivie d'une classification thématique et d'une interprétation des idées du journaliste. Nous montrerons que ce dernier développe un discours complexe sur l'identité juive. En effet, tout en reprenant à son compte les revendications d'acculturation, formulées par les intellectuels et hommes politiques non-juifs de l'époque, il tente de contribuer à l'auto-affirmation des membres de sa communauté.

\section{La presse juive en Allemagne - un moteur dans la formation d'une identité juive moderne}

Forte d'une centaine de titres dans l'espace germanophone avant 1933, la presse juive représente un phénomène important et historiquement ancré (Nagel, 2002 ; MartenFinnis, Winkler, 2006). Sa naissance est intimement liée aux transformations politico-culturelles qui bouleversent le monde juif du XVIIIe siècle. Les premières revues juives voient ainsi le jour en même temps que se développe, autour du philosophe Moses Mendelssohn (1729-1786), la Haskala, le mouvement des Lumières juives (Schulte, 2002 ; Bourel, 2004). Préconisant une sortie de l'isolement de leurs coreligionnaires par le biais d'une éducation " profane », l'initiation à des métiers dits " productifs » et l'apprentissage de la langue allemande, Mendelssohn et ses disciples, les maskilim, se sont en effet efforcés de créer une véritable sphère publique juive, constituée, entre autres, d'un certain nombre de périodiques.

En fondant ces périodiques, les représentants de la Haskala entendent contribuer activement à la formation d'une identité juive moderne. L'objectif de ces publications consistait en effet essentiellement à propager, au sein de la bourgeoisie juive naissante, les idées des Lumières d'une " amélioration civique » (bürgerliche Verbesserung) des Juifs, formulées, entre autres, par le conseiller au ministère prussien de la Guerre Christian Wilhelm von Dohm dans son ouvrage « De l'amélioration civique 
des Juifs» (1781) (Richter, 2010). Le Kohelet Mussar (vers 1755) et Ha-me'assef (1782), tous deux publiés en hébreu, sont parmi les périodiques les plus importants (Toury, 1967 ; Kennecke, 2002). Le choix de l'hébreu peut étonner car l'un des objectifs des maskilim était d'initier leurs coreligionnaires à l'apprentissage de l'allemand. Mais, en publiant dans cette langue, Mendelssohn et ses disciples étaient sûrs de se faire comprendre par les nombreux Juifs ne maîtrisant pas encore l'allemand. Il faut attendre le début du XIXe siècle pour voir apparaître Sulamith (1806), la première revue juive de langue allemande (Lässig, 2004, p. 445-446). Si ses fondateurs choisissent l'allemand comme langue de publication, affichant ainsi clairement leur volonté de créer une forme d'identité judéo-allemande, ils restent, du point de vue du contenu qu'ils véhiculent, fidèles à l'esprit du XVIIIe siècle. A l'instar du Kohelet Mussar et de Ha-Me'assef, Sulamith entend essentiellement « encourager la culture et l'humanité au sein de la nation juive ${ }^{2} »$.

À partir des années 1820, on assiste à une diversification du paysage médiatique juif dans les Etats allemands. Certaines revues s'efforcent de sensibiliser leurs lecteurs aux réformes religieuses, comme la très érudite Wissenschaftliche Zeitschrift für jüdische Theologie (1835-1848) ou le plus populaire Israelitisches Predigt- und Schulmagazin (1834-1836) ; d'autres ont des objectifs plus politiques, comme par exemple le mensuel Der Jude (1832) qui exprime un judaïsme plus affirmé et revendicatif. Rappelons qu'à cette époque, les Juifs allemands ne sont pas encore citoyens au même titre que les non-Juifs. Les droits politiques octroyés suite à l'occupation napoléonienne au début du $\mathrm{XIX}^{\mathrm{e}}$ siècle dans une partie des États allemands ${ }^{3}$ ont été révoqués après le congrès de Vienne et ne seront définitivement acquis qu'au début des années 1860. "Obtenir l'égalité civique » des Juifs allemands constitue donc, pour le fondateur de Der Jude, Gabriel Riesser, l'objectif premier de son journal, et la minorité juive est invitée à jouer un rôle actif dans cette lutte (Der Jude, 10.4.1831, p. 1).

\section{L'exemple de l'Allgemeine Zeitung des Judenthums}

Plus encore que les revues décrites brièvement ci-dessus, l'Allgemeine Zeitung des Judenthums occupe un rôle important dans les processus de formation d'une identité juive moderne. Malgré un tirage ne dépassant guère les 1600 exemplaires, la revue de Philippson était le périodique le plus lu de l'espace germanophone et le premier à s'imposer durablement auprès du public. Sa longévité - le dernier numéro paraît en

2 Voir le sous-titre de cette revue : « Zur Beförderung der Kultur und Humanität unter der jüdischen Nation ».

3 Comme dans le royaume de Westphalie (1808), les grands duchés de Bade (1809) ou le royaume de Prusse (1812). 
1922 - est sans doute due à son style simple, à sa fréquence de parution ${ }^{4}$, à la variété des thèmes abordés et à son important réseau de correspondants nationaux et internationaux autant de caractéristiques qui confèrent à la revue un côté dynamique.

Si le fondateur de l'Allgemeine Zeitung des Judenthums inscrit sa revue dans la lignée de Sulamith et de Der Jude, son projet dépasse aussi ce cadre. À l'instar de ces prédécesseurs, il œuvre, par le biais de son journal, pour « l'amélioration civique » des Juifs allemands et pour leur intégration à la nation allemande mais il s'attache aussi à pérenniser l'identité juive. Pour Philippson, les objectifs définis lors de la fondation de son journal en 1837 et reformulés au fil des ans consistent en effet non seulement à « éveiller (...) le développement de l'esprit » (AZJ, 27.11.1848, p. 698 $)$, à « faire éclater le joug de la restriction, du préjugé et de la haine » (AZJ, 27.7.1837, p. 162) mais aussi à «donner expression (...) à la vie actuelle du judaïsme dans sa globalité (...) pour la réveiller et la faire durer » (AZJ, 4.1.1940, p.1).

\section{3. « Amélioration civique » et intégration}

À bien des égards, Philippson fait siennes les revendications d' "amélioration civique» apparues au cours du XVIIIe siècle. Pour le rabbin allemand, l'évolution devait mener progressivement à une " amalgamation " (Amalgamierung) de la population juive avec la majorité non-juive dans tous les domaines à l'exception de la vie religieuse (AZJ, 1.5.1848, pp. 271-272). Dans l'article programmatique, publié à l'occasion de la sortie du premier numéro, il déclare ainsi très clairement qu'un des objectifs de son journal est l'obtention des droits civiques pour que les Juifs allemands puissent « s'amalgamer avec le peuple allemand " (AZJ, Prospectus, 1937). Pour cela, ils devraient selon lui renoncer à vouloir former un peuple pour n'être plus qu'une communauté religieuse (AZJ, 9.4.1842, p. 210). En effet, l'intégration sociale et politique à la nation est, pour Philippson, intimement liée à une modernisation religieuse du judaïsme (Meyer, 2000). Dans de nombreux articles, il défend des réformes religieuses qui permettraient d'accélérer le passage du judaïsme comme religion-peuple au judaïsme comme simple confession parmi d'autres. Selon lui, il est " nécessaire d'écarter ce qui, dans la forme extérieure de la religion israélite, touche encore au national» (AZJ, 9.4.1842, p. 210). Tantôt il exige de supprimer de la liturgie juive toutes

4 Paraissant d'abord trois fois par semaine, l'Allgemeine Zeitung des Judenthums devient, à partir de 1839, un journal hebdomadaire. II ne s'agit pas d'un journal d'information à proprement parler mais plutôt d'une revue avec de longs éditoriaux et articles qui occupaient Philippson parfois pendant plusieurs semaines. Jusqu'à sa mort en 1889, la revue est essentiellement l'œuvre de son fondateur qui rédige la plupart des textes.

5 Toutes les traductions de l'allemand sont de l'auteur. 
les références à la Palestine et tout ce qui se rapporte de près ou de loin à une restauration nationale : selon lui, " beaucoup de choses dans la synagogue sont restées en l'état qui ne relèvent pas strictement du religieux mais du national » (AZJ, 9.4.1842, p. 210). Tantôt il se prononce en faveur de l'introduction, dans l'office religieux, de l'orgue et du chœur ainsi qu'en faveur de la récitation de quelques prières et d'un sermon hebdomadaire en allemand, afin d'adapter les pratiques religieuses aux normes chrétiennes allemandes.

À la revendication de réformes religieuses fait écho, dans le domaine civil, la revendication d'un changement socio-économique. Comme Ludwig Philippson le souligne à plusieurs reprises dans ses articles, la propension des Juifs à se tourner vers les métiers du commerce et des finances est un héritage de l'oppression, mais à ses yeux, ils doivent désormais s'ouvrir à la formation séculière et se tourner vers l'artisanat et l'agriculture, devenir " productifs », comme le leur demandent les partisans de l'assimilation. Dans un article daté de mai 1848, le rédacteur allemand recommande ainsi expressément à ses lecteurs de s'atteler à la diversification socioéconomique :

"Plus de séparatisme, plus d'exclusion, plus de particularisme! Et ce, surtout dans les relations professionnelles. Parents, vous avez désormais la possibilité de donner un avenir à vos enfants et de les former à ce que vous voulez, et eux ont la possibilité d'accéder à des capacités et au plaisir - alors faites-le, conduisez-les vers toutes les branches, non plus seulement vers le commerce mais aussi vers la science, l'art, l'artisanat, le travail, quel qu'il soit. Et tel qu'il est : sérieux, profond, magistral » (AZJ, 1.5.1848, p. 272).

Un autre aspect essentiel de "l'amélioration civique » prônée par Philippson est l'engagement patriotique des Juifs. Le service militaire et la participation aux guerres étant considérés, au XIXe siècle, comme les principaux signes du sentiment patriotique (Buschmann, 2003), les soldats juifs étaient vus comme des modèles d'émancipation et $d$ ' " amélioration civique ». Leur existence et leur courage remettaient notamment en question les préjugés anti-juifs selon lesquels les membres de la communauté étaient trop timorés, inaptes sur le plan physique ou manquaient de patriotisme pour s'engager dans une lutte armée pour leur patrie. Dans de nombreux articles, Philippson invite non seulement ses coreligionnaires à ne plus demander " s'il y a des Juifs dans l'armée adverse ! " mais à se battre " contre eux » (AZJ, 1.5.1848, p. 272). Pour invalider les stéréotypes anti-juifs cités ci-dessus, il veille aussi soigneusement à ce que la solidarité nationale de ses coreligionnaires soit connue et reconnue par les citoyens non-juifs. Au moment de la Guerre de 1870-1871 entre la France et l'Empire allemand, le publiciste publie ainsi un Livre à la mémoire des Israélites allemands de la Guerre de 1870-1871 où figurent les noms des combattants juifs, classés par ville ou par village, dans le but de « constater la contribution des Juifs allemands à cette mémorable guerre » (AZJ, 20.6.1871, p. 496). Nombreux sont aussi les passages où il exhorte ses coreligionnaires à se comporter en citoyens, comme celui publié en mai 1848, quelques semaines après les événements révolutionnaires qui 
semblent pour un bref instant réaliser l'égalité politique des Juifs d'Allemagne : « Mes frères! (...) Soyez partout des citoyens au sens propre du terme. Le bien du peuple relève autant de notre salut que de celui de l'Etat. Impliquez-vous (...) et aidez selon vos capacités et vos forces. » (AZJ, 1.5.1848, p. 272).

\section{Affirmation identitaire}

De cette manière, Philippson reprend dans son journal bon nombre des revendications d'acculturation de l'époque. L'identité juive ne doit, selon lui, plus être nationale mais seulement religieuse. Cependant, cela ne fait pas de son journal un simple instrument des discours assimilationnistes. Au contraire, dans ses articles, le rabbin allemand contribue également à l'affirmation de certains aspects de l'identité juive. La définition qu'il donne de l'identité juive est intéressante à cet égard. Pour Philippson, si l'identité juive dont il se revendique n'est plus nationale, elle comprend cependant toujours une certaine dimension ethnique ou, comme il le formule luimême, elle est porteuse d'une certaine "individualité ». Dans un article au titre évocateur, "Les particularités des Juifs», il explique qu' " on ne peut pas nier le fait que le peuple juif (...) constitue une particularité, qu'il a une certaine individualité ». Les fondements de cette « individualité » sont la religion mais aussi l'ascendance et le destin communs. Le rédacteur en chef de l'Allgemeine Zeitung des Judentums semble même accepter l'idée d'une physionomie caractéristique comme l'expression de cette appartenance communautaire : " De l'extérieur, cette individualité s'exprime par des traits physionomiques impossibles à méconnaître. Le type juif a marqué le visage et le corps et ne se laisse pas effacer d'un coup d'éponge » (AZJ, 5.11.1849, p. 635).

L'insistance sur « l'individualité juive » ne se fait pas dans une perspective exclusive ou raciste ${ }^{6}$, mais a plutôt pour but de créer un sentiment identitaire au sein d'une communauté juive se désintéressant de plus en plus du judaïsme (Meyer, 1996) et de revendiquer, comme l'a souligné l'historien des Juifs allemands Till van Rahden, « une forme légitime de différence culturelle et ethnique » (van Rahden, 2006) au sein de la société. Selon la vision de Philippson, la société doit autoriser toutes les formes de vie culturelle, intellectuelle et religieuse des Juifs et d'autres minorités, à l'exception de la dimension politique. L'Etat doit en effet " amener l'humanité à la plus grande unité » mais aussi permettre à chaque individu d'atteindre « la plus grande réalisation de [lui]-même » (AZJ, 24.1.1853, p. 55)

6 Fidèle à l'universalisme des Lumières, Philippson s'en prend ainsi régulièrement aux théories de l'Etat chrétien, aux théories raciales et antisémites en vogue à l'époque en leur opposant la diversité des univers d'appartenances dans lesquels s'élabore l'identité individuelle. 
L'" individualité » des Juifs, dans ses dimensions religieuse, culturelle, et aussi ethnique, ne doit donc pas être " éliminée » ou " affaiblie », comme le voudraient certains partisans d'une assimilation des juifs à la société environnante ou les antisémites. Par le biais de son journal, Philippson veut, au contraire, « encourager et renforcer " cette individualité (AZJ, 5.11.1849, p. 634). Afin de " transmettre les particularités » (AZJ, 27.7.1837, p. 161) des Juifs, il prend donc soin de consacrer de nombreux articles à l'histoire et à la culture juive, aux débats religieux et à la vie communautaire dans le but d'informer les lecteurs sur le judaïsme et de préserver ainsi leur attachement à ce dernier. L'indication ajoutée au titre de la revue confirme que sa démarche a une dimension volontairement identitaire : l'Allgemeine Zeitung des Judentums est ainsi « un organe impartial pour tous les intérêts juifs concernant la politique, la religion, la littérature, l'histoire, les langues et les belles lettres ».

Une autre stratégie visant à préserver l'identité juive consiste en l'établissement de liens transnationaux. Grâce à un réseau de correspondants internationaux et à la publication d'articles ou de commentaires de confrères étrangers, l'Allgemeine Zeitung des Judenthums apparaît comme un véritable lieu d'échanges par-delà les frontières. Philippson revendique d'ailleurs cette fonction de trait d'union de son journal. Il souligne que son journal a largement contribué à la création d'un " esprit commun » (AZJ, 24.12.1861, p. 746), en formant un « lien entre ceux qui, dispersés sur tous les continents, se réclament de notre religion » (AZJ, 2.8.1879, p. 611). A travers l'échange transfrontalier avec des Juifs d'autres pays - notamment lorsque l'identité juive est fragilisée, remise en cause ou menacée - , Philippson semble trouver un point d'appui rassurant, lui permettant de consolider l'identité juive en Allemagne (Kastoryano, $2007)^{7}$. On peut ainsi observer que Philippson tourne souvent son regard vers la France pour justifier l'obtention de l'égalité politique ou pour combattre l'antisémitisme (Knörzer, 2013).

\section{Conclusion}

Si le rôle de la presse juive dans les processus de restructuration de l'identité juive au cours du "siècle de l'émancipation " a longtemps été négligé, l'historiographie a récemment vu paraître des études insistant sur le lien entre presse et identité juive moderne (Lässig, 2004 ; Neumann-Schliski 2011). Dans ces analyses, la presse juive et ses rédacteurs apparaissent comme de véritables acteurs de la restructuration identitaire des juifs. Dans la continuité de ces recherches, cet article montre que

7 Kastoryano souligne notamment l'apport psychologique des relations et activités transnationales des minorités. Les migrants, souvent dans des situations d'exclusion ou de stigmatisation, trouveraient ainsi un point d'appui dans les réseaux transfrontaliers, leur permettant à la fois d'affirmer et de consolider leur identité. 
Philippson et son journal, l'Allgemeine Zeitung des Judenthums, constituent un bel exemple d'une telle restructuration. Dans sa revue, le rabbin allemand tente non seulement de valoriser la tradition juive, mais il revendique également l'expression de cette dernière au sein d'une société qu'il voudrait ouverte à la différence, prenant ainsi le contre-pied des discours nationalistes, racistes et antisémites en vogue. Et même lorsque Ludwig Philippson cherche à adapter le judaïsme à la société environnante, l'Allgemeine Zeitung des Judenthums ne peut être réduit à un simple instrument des revendications d'acculturation formulées par les intellectuels et hommes politiques non-juifs de l'époque car ce dernier participe activement au " projet juif de modernisation » qui se développe au XIXe siècle et dont le résultat a été qualifié de « réinvention d'une tradition » (Volkov, 2001).

L'engagement fort de Philippson, en faveur selon ses termes du « judaïsme tout entier, sa vivification, son redressement et sa justification » (AZJ, 4.1.1840, p. 2), caractérise aussi d'autres domaines de sa vie. Philippson est ainsi à l'origine d'un certain nombre de pétitions combattant l'exclusion de Juifs allemands. En 1854, il crée l'Institut pour la Promotion de la Littérature israélite, le premier club du livre juif et il joue un rôle considérable dans l'avènement de la Hochschule für die Wis-senschaft des Judenthums, la première faculté juive d'Allemagne qui est fondée en 1872 à Berlin. Ces initiatives ainsi que son activité journalistique visent davantage que l'intégration des Juifs allemands à la société environnante. L'aspiration émancipatrice et modernisatrice se combinent chez lui à un profond désir d'assurer activement la survie du judaïsme, de sorte que décrire Philippson comme un véritable " défenseur et combattant d'Israël » (AZJ, 1.1.1890, p. 5), comme le fait son successeur Gustav Karpeles dans sa nécrologie, semble plus que justifié.

\section{Bibliographie :}

ASCHHEIM, S. (1982) : Brothers and Strangers. The Eastern Europe-an Jew in German and German-Jewish Consciouness. Madison, Wisconsin : University of Wisconsin Press.

BARDIN, L. (1996) : L'analyse du contenu. Paris : PUF.

BOUREL, D. (2004), Moses Mendelssohn. La naissance du judaïsme moderne. Paris : Gallimard.

BUSCHMANN, N. (2003) : Einkreisung und Waffenbruderschaft. Die öffentliche Deutung von Krieg und Nation in Deutschland 1850-1871. Göttingen : Vandenhoeck, Ruprecht.

KASTORYANO, R. (2007) : « Participation transnationale et citoyenneté : les immigrés dans l'Union européenne ». In Cultures et conflits, $\mathrm{n}^{\circ} 28$, hiver 1997, mis en ligne le 21 mars 2007, consulté le 1 novembre 2014, 〈http://conflits.revues.org/2121〉. 
KENNECKE, A. (2002) : « Der Ha-Me'assef und sein erster Herausgeber Isaac Euchel » In NAGEL, M., ed (2002): Zwischen Selbstbehauptung und Verfolgung. Deutschjüdische Zeitungzn uns Zeitschriften von der Aufklärung bis zum Nationalsozialismus. Hildesheim, Zürich, New York: Georg Olms Verlag, pp. 67-83.

KNÖRZER, H. (2013) : « La presse juive comme espace politique transnational au XIXe siècle ». In Archives juives. Revue d'histoire des Juifs de France, $\mathrm{n}^{\circ}$ 2, pp. 81-95.

LÄSSIG, S. (2004) : Jüdische Wege in Bürgertum. Kulturelles Kapital und sozialer Aufstieg im 19. Jahrhundert. Göttingen : Vandenhoeck, Ruprecht.

MARTEN-FINNIS, S., WINKLER, M., ed (2006) : Die jüdische Presse im europäischen Kontext 1668-1990. Brême : Editions Lumière.

MARTEN-FINNIS, S., WINKLER, M., ed (2006): Die jüdische Presse. Forschungsmethoden, Erfahrungen, Ergebnisse. Brême : Edi-tions Lumière.

MEYER, M.A. (1996), « Jüdische Gemeinden im Übergang », In BRENNER, M. et al., ed (1996) : Deutsch-jüdische Geschichte der Neuzeit, 1780-1871, tome 2. Munich : Beck. pp. 96-134.

MEYER, M.A. (2000): Antwort auf die Moderne. Geschichte der Reformbewegung im Judentum. Vienne, Cologne, Weimar : Böhlau.

NAGEL, M., ed (2002): Zwischen Selbstbehauptung und Verfolgung. Deutsch-jüdische Zeitungzn uns Zeitschriften von der Aufklärung bis zum Nationalsozialismus. Hildesheim, Zürich, New York : Georg Olms Verlag.

NEUMANN-SCHLISKI, J. (2011), Konfession oder Stamm? Konzepte jüdischer Identität bei Redakteuren jüdischer Zeitschriften 1840 bis 1881 im internationalen Vergleich. Brême : Editions Lumière.

RAHDEN, T. van (2006) : "Germans of the Jewish Stamm. Visions of Community between Nationalism and Particularism, 1850-1933 " In GREGOR, N., ROEMER, N., ROSEMENN, M., ed, (2006) : German History from the Margins, Bloomington, Indianapolis: Indiana Univer-sity Press. pp. 27-48.

RICHTER, S. (2010) : « L'émancipation des juifs en Prusse et en France au XVIIIe siècle, un discours européen ? " In KNÖRZER, H., ed. (2010), Expériences croisées. Juifs de France et d'Allemagne aux XIXe et XXe siècle. Paris : Editions de l'Eclat.

SCHULTE, Ch. (2002): Die jüdische Aufklärung. Philosophie, Religion, Geschichte. Munich : Beck.

TOURY, J. (1967) : « Die Anfänge des jüdischen Zeitungswesens in Deutschland », In Bulletin des Leo Baeck Instituts, vol 38-39, pp. 93-123. 
VOLKOV, S. (2001) : « Die Erfindung einer Tradition. Zur Entstehung des modernen Judentums in Deutschland. ", In VOLKOV, S. (2001): Das jüdische Projekt der Moderne, Munich : Beck. pp. 118-137. 